\title{
Create a Virtual Mannequin Through the 2-D Image- based Anthropometric Measurement and Radius Distance Free Form Deformation
}

\author{
Sheng-Fuu Lin \\ Institute of Electrical Control Engineering \\ National Chiao Tung University \\ Hsinchu, Taiwan(ROC)
}

\author{
Shih-Che Chien \\ Institute of Electrical Control Engineering \\ National Chiao Tung University \\ Hsinchu, Taiwan(ROC)
}

\begin{abstract}
D human body models are used in a wild spectrum of applications, such as film and entertainment industry, that require images of human replicas, but the computer generated models of human body generally do not adequately model the complex human morphology. These models do not reflect the realistic anthropometric data and are not specific enough for commercial use. This paper presents an approach to adjust virtual mannequins through the use of anthropometric measurement data, which are obtained from 2-D image-base measurement. In this approach, a novel method which used the Chinese medicine acupuncture theory for fast position locating and human body slice model to approach circumferences is proposed to 2-D image-based anthropometric measurement. The measurement data are used in grouping 3-D scanned body objects into clusters. The virtual mannequins are then adjusted by using the measurement data of the standard model that belong to its cluster. In this way, the realistic accurate virtual mannequins are created.
\end{abstract}

Keywords-human body; anthropometric measurement; free form deformation deformation

\section{INTRODUCTION}

3-D human body models are used in a wild spectrum of applications, such as film and entertainment industry, that require images of human replicas. In film and entertainment industry, the 3-D human body models, virtual mannequin, are employed in visualization and animation environment. Although there are many techniques in 3-D virtual environment, depending on the functionalities of each element, efficient shading, skinning, and motion algorithms, to simulate the physical properties, most of these techniques are focus on human body models. Because of it can be used to create the personalized virtual mannequins and imitate the movement of human. Internet applications such as 3-D games and advertisement videos contain personalized human body model. In particular, computer generated models of human body usually do not adequately model the complex human morphology and stand for a lot of applications.

The method used for creating or adjusting virtual human body model has been evolved. Two major steps, anthropometric measurement and adjustable virtual mannequin, are always utilized for creating the personalized 3-D human body model. The anthropometric measurements are provided the personalized body measurement data, such as height, chest circumference, waist circumference...etc, for mannequin adjustment. The adjustable virtual mannequin model provides a platform for adjusting 3-D human body model to approach the realistic body.

Anthropometry is a since of measurements which is used in order to establish the physical geometry, mass property, and strength capabilities of the human body [1]. In traditional anthropometric measurement methods, the measurement can be done by using a simple instrument such as tape and without complex measurement pre-setting [2]. Although the traditional anthropometry measurement is easy and convenient to use, the traditional measurement relies on manual operations that are inefficient and prone to errors. 2D image-based anthropometric measurement methods adopt two or more photographic, and through image process and geometric transformation, the anthropometric measurement data can be obtained from captured images. Meunier and Yin [3] proposed an anthropometric measurement system that can generate body measurements from two-dimension images. Hung et al. [4] used geometric shapes (ellipse and rectangular) to approximate the shape of critical part circumference. The ellipse shape was used to approximate neck, wrist, and palm circumferences, and the major and minor axes length were obtained from the front and side views.

Nowadays, the 3-D laser scanning technique makes it possible to digitalize the complete surface of human body and provides much richer information about the body shape than the traditional anthropometric measurements [5]. CAESAR, which stands for Civilian American and European Surface Anthropometry Resource, is the first large scale 3-D anthropometry survey project [6]. This approach provides a standard model of digitalized human body shape and opens up opportunities to extract new measurements for quantifying the body shape. The first attempt in processing 3-D anthropometric data for analyzing the body shape is to extract traditional anthropometric measurements from the scanned data [7]. Although working with the 3-D surface data has the advantage of being able to perform repeated measurements without the subject being present, the 3-D scanning equipment is not available in anytime and anywhere and the collected 3-D scanning data still needs complicated analysis to calculate human body anthropometric data. 
The creating methodologies of 3-D human body can be divided into three parts, 3-D scanning, reconstruction, and example based [8][9]. The 3-D scanning method is described in past paragraph, which used precise optoelectronic measurement device for body shape scanning. In unknown human body model creating, the example based method is appropriated. In this paper, we concern about how to create virtual mannequin that more approaching realistic and saving more processing time.

The most of example based methods are adopted the space deformation methods. The space deformation method is the process of mapping some of vertices from one space to the other. Because of space mapping methodology, the wide range deformation of 3-D object can be developed. Barr's [10] presented a work on parametric shape and Scheeper's [11] is also used the parametric shape for modeling human body muscle. Sederberg and Parry proposed the Free Form Deformation (FFD) method that embedded the 3-D object into a cubic box, which had several control points to influence vertices of object, deforming the box will also deform the underlying object. Borrel [12] proposed a variant FFD for different type of object parts.

The adjustment of virtual human body model to fit real measurement data provides an important challenge to the research community. This paper presents an approach that adopts the benefit and avoids the disadvantage of 3-D scanned human model. The 2-D image-based anthropologic measurement [13], that using Chinese medicine acupuncture theory for position locating and human body slice model for circumference approaching, is applied for body measurement. In this approach, we propose a method Radius Distance Free Form Deformation (RD-FFD) for geometric deformation. The Radius Distance system is a coordinate in polar coordinate system $(r, \theta)$. Basically the radius distance is the scalar Euclidean distance between a point and origin of the system of coordinates. In RD-FFD system, the model divided into slices and a radius of each slice is scaled to achieve the deformation effect to instead of using polygonal methods. Since the deformation scales are along with the radius direction in radius distance system, the detail of human body deformation could be exhibited and help to save more computing time.

This paper is organized as follows. Section 2 provides a $2 \mathrm{D}$ image-based anthropometric measurement that used two side images, front and side, which captured by camera, and the human body slice model is supplied to approximate the critical circumference shape. In section 3, the method Radius Distance Free Form Deformation (RD-FFD) for geometric deformation is proposed. The experimental results and discussion are showed in section 4. Section 5 concludes this paper. The flowchart of this system illustrated in Fig. 1.

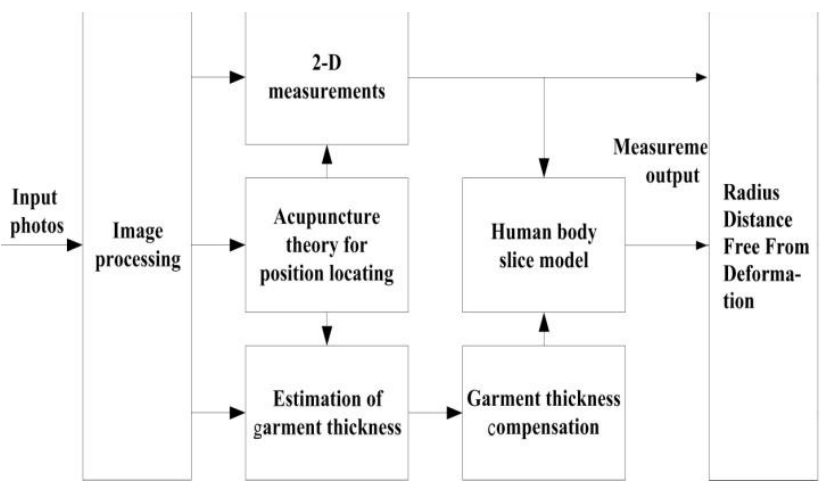

Figure 1. The flowchart of this approach

\section{2D IMAGE-BASED ANTHROPOMETRIC MEASUREMENT SYSTEM}

The method of 2-D image based anthropometric measurement includes 2-D image preprocessing, Chinese medicine acupuncture theory for position locating, and human body slice model. At the first, the image preprocessing is used to process the images captured from camera. Secondly, the Chinese medicine acupuncture theory is adopted to locate the critical position because of its distinctive acupuncture point locating method of human body. After locating the critical position, the 2-D anthropometric data can be obtained by direct measurement. In order to acquire the circumference, the human body slice model is used to approximate the circumference shape. At the last, an efficient compensation system that aims to reduce the influence of subject wearing clothes for measurement is presented.

\section{A. 2-D image preprocessing}

The camera is set on tripod with about one meter off the ground, and dual-axis bubble levels on tripod help to keep the camera level. The system captures front and side images of objects standing with some required postures, as show in Fig. 2(a). The extraction of human body shape is performed by skin color extraction and automatic image segmentation [14][15], which uses color edge extraction and seeded region growing (SRG) to do image segmentation. Then, the full body silhouette shape can be obtained, as show in Fig. 2(b).

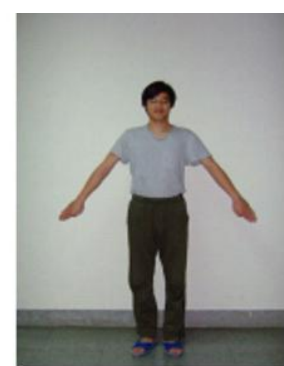

(a)

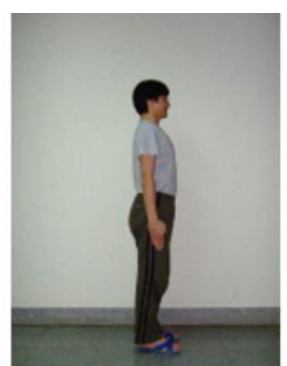

(b)

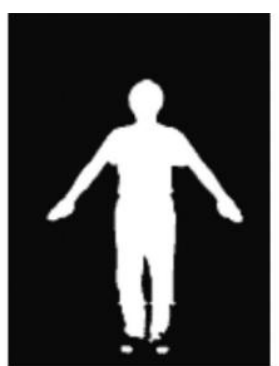

(c)
Figure 2. (a)(b) The postures of front and side view. (c) The human body segmentation of front view image. 


\section{B. Chinese medicine acupuncture theory for position locating}

Acupuncture is the procedure of inserting filiform needles into various acupuncture points on the body to relieve pain or for therapeutic purposes. In treatment procedural, the therapist should find out the correct acupuncture point position corresponding to pain before inserting needle [16]. Hence, the acupuncture point location method is developed to locate the acupuncture points on human body fast and accurately. Five body measurement data (shoulder length, chest circumference, waist circumference, hip circumference, and leg length) are selected for discussion because there are the most commonly used in manufactory and general application, such as clothing size. For locating the corresponding position, six acupuncture points (Lian-Quan, Tian-Tu, Chien-Yu, Shan-Zhong, Shen-Que, and $\mathrm{Qu}-\mathrm{Gu}$ ) are taken to locate the critical positions and show as Fig. 3.

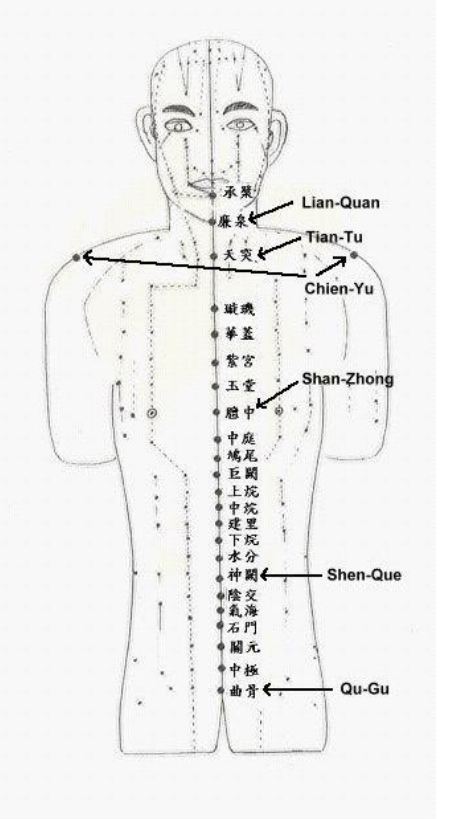

Fig. 3 Illustrate the position of acupuncture points on human body.

The measurement data can be separated into two groups, direct measurement (linear) and indirect measurement (circumference). In linear part, the measurement is to calculate the distance between two acupuncture points: shoulder length is the distance between two Chien-Yu acupuncture points or to calculate the distance between the acupuncture point and extremity: leg length is the distance between the Qu-Gu acupuncture point and sole of foot. Circumference cannot be measured directly using front and side view images only and must therefore be calculated using some form of mathematical model. In this paper, the human body slice model is adopted to approximate the circumference shape, and the circumference could be obtained through the calculation with two parameters, the corresponding width of front and side view image.

\section{Human body slice model}

Circumference cannot be measured directly using only 2D measurement, and therefore the mathematical model is applied to approximate the corresponding circumference. In this paper, the human body slice model which using Bezier curve [17] is used to approximate the circumference. In this paper, the cubic Bezier curve is adopted for basic curve to patch the piecewise curve. The cubic Bezier curve content with four control points: $\mathrm{P} 0, \mathrm{P} 1, \mathrm{P} 2$, and $\mathrm{P} 3$, which existing on the same plane of curve. The cubic Bezier curve $\mathrm{B}(\mathrm{t})$ starts from $\mathrm{P} 0$ to $\mathrm{P} 1$ and arrive at $\mathrm{P} 3$, and the function can be denoted as follow:

$$
\begin{aligned}
B(t) & =(1-t)^{3} P_{0}+3(1-t)^{2} t P_{1}+3(1-t) t^{2} P_{2}+t^{3} P_{3}, t \in[0,1] . \\
& =\left[\begin{array}{lllll}
t^{3} & t^{2} & t & 1
\end{array}\right]\left[\begin{array}{cccc}
-1 & 3 & -3 & 1 \\
3 & -6 & 3 & 0 \\
-3 & 3 & 0 & 0 \\
1 & 0 & 0 & 0
\end{array}\right]\left[\begin{array}{l}
P_{0} \\
P_{1} \\
P_{2} \\
P_{3}
\end{array}\right] \\
& =T B_{c} P
\end{aligned}
$$

The 3-D human body scanning data is the body digitalized data, and the slice data can be extracted with the plane which is concerned. The Taiwan human body bank (TAIBBK) [18][19], which stands for Industrial Technology Research Institute, Tshing Hua University, and Chang Gung University, is a large scale 3-D anthropometric measurement project. About 1100 civilians, between the age of 19 and 65 in Taiwan, were scanned. In generally, the shape of human body is virtually bilateral reflection symmetry with respect to the center mirror plane. Furthermore, the shapes of chest, waist, and hip are also symmetrically in slice human model. It also means that the circumference can be obtained, if the curve length of corresponding half-shape is known. Fig. 4(a) shows the whole and half chest slice shape of standard male build type, and confirms the symmetry of slice shape model. the piecewise polynomial curve based on cubic Bezier curve is used to approximate the curve of half-shape, and then the curve length can be calculated by using definite integral. Fig. 4(b) shows the curve approximation with two cubic Bezier curves, and two sets of control points are marked with blue circle and star. The red curve is actual curve of chest slice shape and the blue dotted curve is the approximation curve, which is similar to manual measurement.

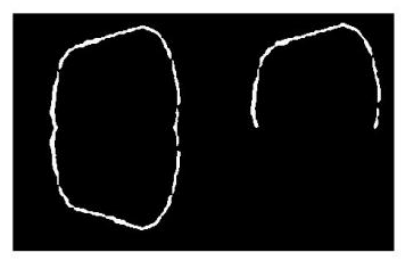

(a)

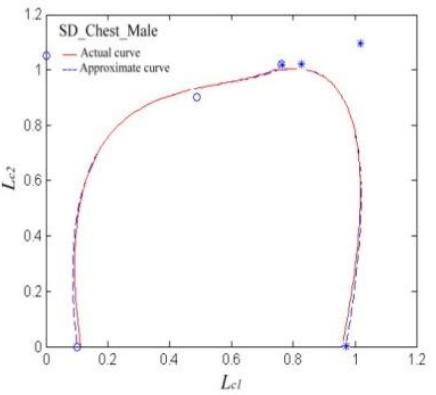

(b)
Figure 4. (a) The whole and half chest shape of standard male build type. (b) The approximation curve and actual curve of chest

Then, the expression of piecewise polynomial curve can be denoted as follow:

$$
C(t)=C_{b z 1}+C_{b z 2}=T B_{c}\left(P_{1}+P_{2}\right)
$$

where $\mathrm{P} 1$ and $\mathrm{P} 2$ are the control point sets of anterior curve $(\mathrm{Cbz} 1)$ and posterior curve (Cbz2) respectively. Now, we take 
the chest circumference measurement for example. The expression of approximation curve, showed in Fig. 3(b) and followed from Eq. (4) is showed as:

$$
\begin{gathered}
C_{\text {chest }}(t)=T B_{c}\left(P_{c 1}+P_{c 2}\right) \\
p_{c 1}=\left[\begin{array}{cc}
0.1 & 0 \\
0 & 1.05 \\
0.49 & 0.88 \\
0.75 & 1
\end{array}\right]\left[\begin{array}{cc}
L_{c 1} & 0 \\
0 & L_{c 2}
\end{array}\right] \\
P_{c 2}=\left[\begin{array}{cc}
0.75 & 1 \\
0.82 & 1 \\
1.14 & 1.11 \\
0.97 & 0
\end{array}\right]\left[\begin{array}{cc}
L_{c 1} & 0 \\
0 & L_{c 2}
\end{array}\right]
\end{gathered}
$$

where $L_{c 1}$ and $L_{c 2}$ are the width of chest in side image and half width of chest in front image respectively.

\section{The compensation system for measurement}

The compensation system provides an efficient method to avoid the error calculation which including garment thickness, therefore the subject has been required for no-wearing clothes or only wearing light underwear in measurement. Two process steps, garment thickness estimation and measurement compensation, are adopted for garment thickness compensation. Our approach is based on the method Lin [13] proposed efficient garment thickness compensation strategy. The fuzzy inference system is used to infer the thickness of garment and the back-propagation neural network (BPNN) is used to structure compensation system.

\section{VIRTUAL MANNEQUIN DEFORMATION}

The Radius Distance system is a polar coordinate system. Basically the radius distance is the scalar Euclidean distance between a point and origin of the system of coordinates. Therefore, the complexity of human body object surface construction and manipulation can often be transferred to sweep-based surface model. The sweep-based surface has many merits in shape deformation and animation of arbitrary $3 \mathrm{D}$ objects. Human body object is especially adequate in sweep-based surface, because the human torso and limbs exhibit a cylindrical topology and the underlying skeleton provides natural axes.

For more precision deformation, the human body object will be separated into several parts, left upper arm, left lower arm, right upper arm, right lower arm, chest part, waist part, hip part, right thigh, right shank, left thigh, and left shank, belong to the major joints. Through sweep-based surface and segmentation, the each contour is attached to the proximal joint and defined in the local coordinate system. For connecting each part, we construct wire frame of each part to connect to neighbor parts, and then we can join them together after deformations of each part. It is clear to observe that the wire in wire frame is the skeleton of human body. Fig. 5 (a) shows the each segmented part of human body and Fig. 5(b) shows the weep-based surface with the wire frame and the major joints are showed as solid blue circles.
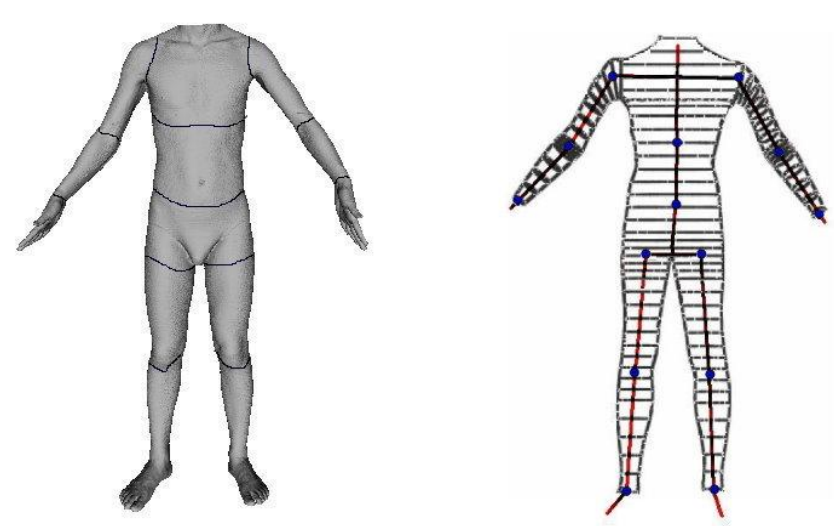

Figure 5. (a) shows the body segmented of human parts and (b) shows the weep-based surface with the wire frame

In this approach, the measurements Body Mass Index (BMI) is used in grouping 3-D body scans, which are obtain using the precise optoelectronic laser scanning measurement device from TAIBBK that described in past paragraph. The virtual mannequins are adjusted by using the measurement data of standard mannequin model. For rough separated of different type of builds, there are 120 human body objects (50 males and 50 females) were selected in TAIBBK database, and the males and females were divided into six clusters on the basis of BMI $(\mathrm{BMI}<18.5,18.5 \leq \mathrm{BMI}<22.5,22.5 \leq \mathrm{BMI}<25,25 \leq \mathrm{BMI}<30$, $30 \leq \mathrm{BMI}<35$, and $\mathrm{BMI} \geq 35$ ) respectively. Each cluster chooses one standard model for represented and deformation to create new mannequin at same cluster.

\section{A. Radius Distance Free Form Deformation}

In this approach, we extend the deformation method, Cylinder Free Form Deformation (CFFD), by using a constrain region instead of a constraint control point and used a novel deformation function to simplify the position computing. The radius distance coordinate contained with the scalar distance between a point and origin (or center) and the azimuth angle between the reference direction and the line from the origin is applied, and it is suitable for Cylinder Free Form Deformation. Fig. 6 is illustrated the cylinder coordinate and Radius Distance Free Form Deformation, and shows the $s$ layers of deformation control (blue circles).

In each layer, the control point are unified distribute with the same angle between the neighbors on the circle contour. The control point $P_{i j}$ is defined as the $i$-th layer and at the $j$-th control point. In our approach, the number of control point on each layer is fixed for 8 points and the cross section point of contour and y axis is assigned for $P_{i 0}$. The radius distance of each control layer is defined as $R$ and the distance between each layer is fixed as $h$, so the height $\mathrm{H}$ of local deformation part is $(s-1) h$. The slices of object are show as red circles and the vertexes are denoted as solid red circle. The radius distance $r_{p q}$ of vertex $v_{p q}$ is defined as the distance between the point $c_{p}$, which is the cross section point of axis and slice, and vertex $v_{p q}$. 


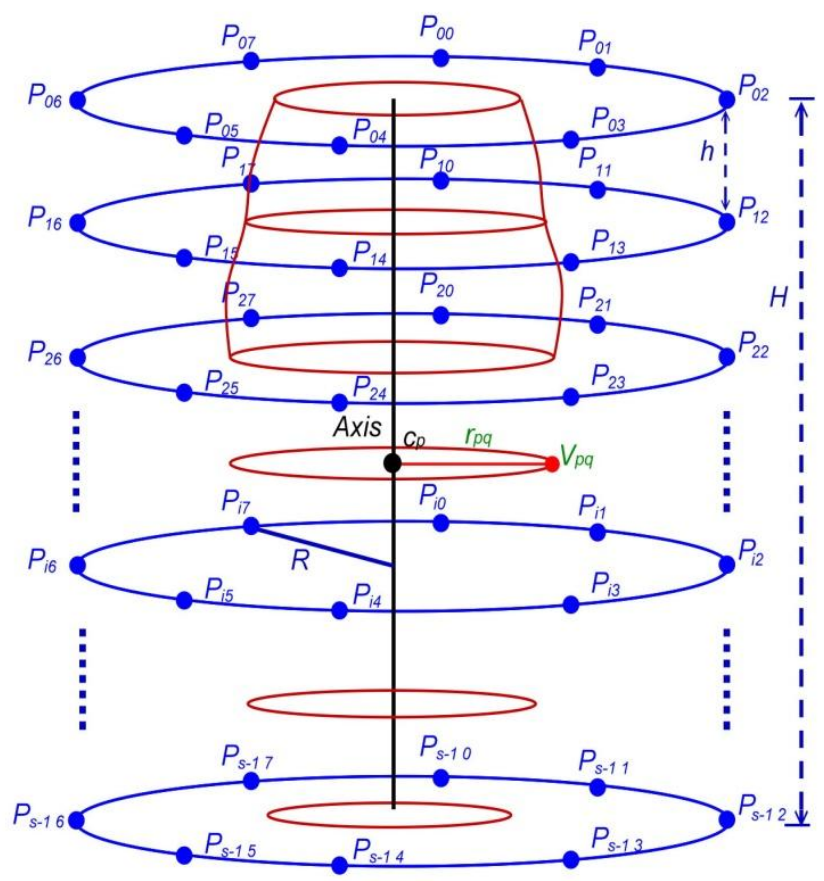

Figure 6. Illustrate the cylinder coordinate and Radius Distance Free Form Deformation.

In sweep-based surface, the object was cut into $n$ slices and the vertex $v_{o q}$ belong to these slice is denoted as the $p$-th layer and $q$-th vertex. The point $v_{o q}$ in cylinder coordinate can be written as:

$$
\begin{aligned}
v_{p q} & =\left(r_{p q}, \theta_{q}, h_{p}\right) \\
& =\left(r_{p q}, \frac{2 q}{m} \pi, p * h\right) \quad p \in[0, n-1] \& q \in[0, m-1]
\end{aligned}
$$

where $m$ is the number of chosen vertexes in $p$-th layer.

For simplified calculation of parametric set of deformation, we choose the vertex locate on contour and have the same azimuth angle with control point. So the number of chosen vertex in each sweep layer is also 8 in training model. Fig. 7 shows the $p$-th slice contour and $i$-th control plane and designated as red and blue circles respectively. The inner red circle is the contour of standard model and outer circle is the contour of training data, and then the movement $\delta_{v_{p q}}$ of vertex $v_{p q}$ can be calculated as:

$$
\vec{\delta}_{v_{p q}}=v_{p q}^{\prime}-v_{p q}=\left(r_{p q}^{\prime}-r_{p q}\right) \overrightarrow{u_{q}} /\left|\overrightarrow{u_{q}}\right|
$$

where $\overrightarrow{u_{q}}$ is the unit vector of $q$-th direction.

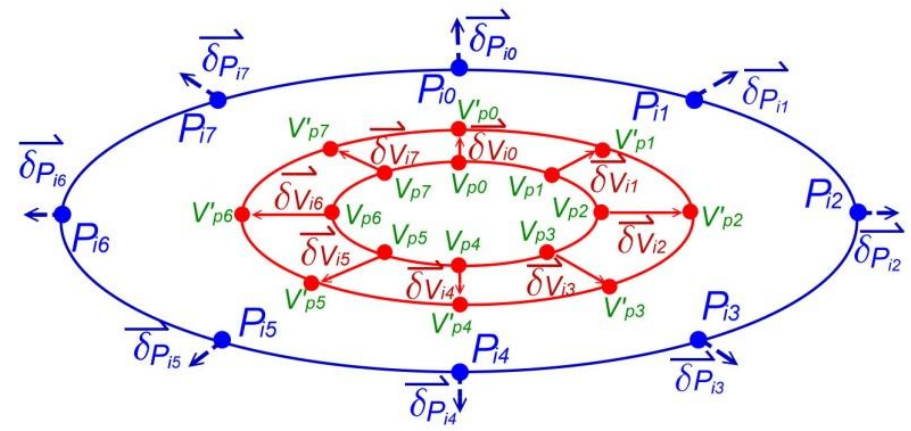

Figure 7. Illustrate the Cylinder Free Form Deformation.

In this paper, we adopt an example-based deformation method that adjusted by using the measurement data of standard mannequin model. So the parametric sets of free form deformation are obtained by training of each cluster that will be done at the beginning. In training model, the function of control point $P_{i j}$ movement is defined as:

$$
\begin{aligned}
\overline{\delta_{P_{i j}}} & =\sum_{p=0}^{n-1} \sum_{q=0}^{m-1} f\left(v_{p q}\right)=\sum_{p=0}^{n-1} \sum_{q=0}^{m-1} D_{i j}(p, q) E_{i j}(p, q) * \overrightarrow{u_{j}} \\
& =\left(C_{r}-1\right) a_{i j} e^{\left|1-C_{r}\right|} * \overrightarrow{u_{j}}
\end{aligned}
$$

where $D_{i j}(p, q)$ is a volume of the distance ratio of vertex $v_{p q}$ to control point $P_{i j}$ and can be written as :

$$
D_{i j}(p, q)=1-\frac{d\left(v_{p q,} P_{i j}\right)}{D_{\max }}
$$

where $d\left(v_{p q}, P_{i j}\right)$ is the distance between $v_{p q}$ and $P_{i j}$ and can be denoted as follow

$$
\begin{aligned}
d\left(v_{p q}, p_{i j}\right)= & {\left[\left(R \sin \theta_{j}-r_{p q} \sin \theta_{q}\right)^{2}+\left(R \cos \theta_{j}-r_{p q} \cos \theta_{q}\right)^{2}\right.} \\
& \left.+((i-1) h-(p-1 / n-1) H)^{2}\right]^{1 / 2} .
\end{aligned}
$$

The definition of $D_{\max }$ is the longest distance in this deformation part and can be assigned for the distance between two control points which belong to top and down plane and has the difference azimuth angle $\pi$.

$$
D_{\max }=\left[(2 R)^{2}+H^{2}\right]^{1 / 2}=\left[(2 R)^{2}+((s-1) h)^{2}\right]^{1 / 2} .
$$

The formula $E_{i j}(p, q)$ is the effective component of vertex $v_{p q}$ to control point $P_{i j}$, hence the $E_{i j}(p, q)$ can be explained for the inner product of the vector of vertex $v_{p q}$ movement to the unit vector of control point $P_{i j}$. The formula $E_{i j}(p, q)$ is showed as:

$$
E_{i j}(p, q)=\overline{\delta_{v_{p q}}} \bullet \overrightarrow{u_{i j}}=\left(v_{p q}^{\prime}-v_{p q}\right) \bullet \overrightarrow{u_{j}}
$$

where $\overline{\delta_{v_{p q}}}$ is delta vector of vertex $v_{o q}$ from standard model 
contour to trainning model contour. $\overrightarrow{u_{j}}$ is the unit vector of $j$ th control point. In the equation (8), $f$ is deformation function where $f: R^{3} \rightarrow R^{3}$ and $a_{i j}$ is the free form deformation parameter set of control point $P_{i j}$. $\mathrm{C}_{\mathrm{r}}$ is the circumference ratio of standard model and training model, and defined as:

$$
C_{r}=L / L_{s t d}
$$

where $L$ is the circumference of training, and $L_{s t d}$ is the circumference of standard model.

The parametric set $A$ of control point can be acquired from (8) and be denoted as:

$$
P=L\left(C_{r}\right) * A * U=\left(C_{r}-1\right) e^{\left|1-C_{r}\right| * A * U}
$$

where $U$ is the unit vector set of each orientation.

After each cluster trained, the parametric sets of each segmented parts are attained. Furthermore, the complete parametric sets of each cluster are obtained through training each cluster. Therefore, the virtual mannequins can be created just only by anthropometric measurement data and Radius Distance Free Form Deformation. The deformation (creation) function of RD-FFD is similar as function (8) and be defined as:

$$
\begin{aligned}
\overline{\delta_{v_{p q}}} & =\sum_{i=0}^{s-1} \sum_{j=0}^{m-1} G\left(p_{i j}\right)=\sum_{i=0}^{s-1} \sum_{j=0}^{m-1} D_{p q}(i, j) E_{p q}(i, j) * \overline{u_{p}} \\
& =v_{p q_{-} s t d}-v_{p q_{-} d e f}
\end{aligned}
$$

where $v_{p q \_s t d}$ is the position of vertex $v_{p q}$ on standard model and $v_{p q_{-} \text {def }}$ is the position of vertex $v_{p q}$ after deformation.

Deforming a segment without any filtering stage will result in discontinuous passed at the boundaries of the regions. The cosine-tape window filters are performed on the boundary vectors of each segment. Then, the overlap and discontinuous vector will be bounded in a sensible value to smooth the conjunction surface.

\section{EXPERIMENTAL RESULTS AND DISCUSSION}

This section describes the experiment results including the accuracy of 2-D image-based anthropometric measurement and demonstrates of RD-FFD by anthropometric measurement. The accuracy part adopts statistic methods to calculate the correlations between proposed system and traditional measurement system. The precision analysis is designed to repeated measurements by proposed and manual method, and aimed to show the repeatability of system.

\section{A. Accuracy}

The accuracy of the 2D image-based measurement system was calculated by comparing 2D image-based measurement with the manual measurement, or called traditional method, taken by anthropometric [20]. The test sample composed of 155 subjects (87 males and 68 females) that have worn short sleeve T-shirt and been measured both with traditional method and with 2D image-based method. Each subject was measured once by each method. The Pearson correlation coefficients between manual and 2D image-based measurements also list in TABLE I. The error rate $(\mathrm{E})$ is the absolute value of difference of measurement and actual value (manual measurement) against the actual value. It is computed using the follow equation

$$
E=\frac{\left|x_{\text {measurement }}-x_{\text {actual }}\right|}{x_{\text {actual }}} \times 100 \%
$$

TABLE I. ACCURACY RESULTS OF SIMPLEX GARMENT (SHORT SLEEVE T-SHIRT)

\begin{tabular}{|rcccc|}
\hline Measurement $(\mathbf{m m})$ & Males & $(\boldsymbol{n}=\mathbf{8 7})$ & Females & $(\boldsymbol{n}=\mathbf{6 8})$ \\
\cline { 2 - 5 } & Corr. & $\boldsymbol{E}_{\text {avg }}$ & Corr. & $\boldsymbol{E}_{\text {avg }}$ \\
\hline Shoulder length & 0.92 & $2.13 \%$ & 0.97 & $1.98 \%$ \\
Chest circumference & 0.94 & $1.79 \%$ & 0.96 & $1.83 \%$ \\
Waist circumference & 0.96 & $1.75 \%$ & 0.97 & $1.72 \%$ \\
Hip circumference & 0.96 & $1.85 \%$ & 0.95 & $1.86 \%$ \\
Leg length & 0.94 & $1.86 \%$ & 0.94 & $1.89 \%$ \\
\hline
\end{tabular}

The test sample composes with 17 males and 11 females and be required to wear 4 kinds of garment, short T-shirt, long T-shirt, thin jacket, and thick jacket, for measurement. In order to assess the accuracies of 2D image-based measurement for each type of garment, the subjects were measured once with each type of garment for $2 \mathrm{D}$ system measurement and once with short sleeve T-shirt for manual measurement. The results of average error rate and Pearson correlation coefficients are listed in TABLE II.

TABLE II. ACCURACY RESULTS OF MANIFOLD GARMENT

\begin{tabular}{|ccccc|}
\hline $\begin{array}{c}\text { Measurement(mm) } \\
(\boldsymbol{n}=\mathbf{4 6})\end{array}$ & \multicolumn{5}{c|}{ Corr. } & $\boldsymbol{E}_{\text {avg }}$ \\
\cline { 2 - 6 } & Corr. & $\boldsymbol{E}_{\text {avg }}$ & \multicolumn{2}{c|}{ Hip circumference } \\
\hline & \multicolumn{2}{c}{ Shoulder length } & 0.96 & $1.88 \%$ \\
2-D system I & 0.91 & $1.92 \%$ & 0.95 & $1.93 \%$ \\
2-D system II & 0.92 & $1.93 \%$ & 0.92 & $1.77 \%$ \\
2-D system III & 0.91 & $2.11 \%$ & 0.93 & $2.09 \%$ \\
2-D system IV & 0.90 & $2.29 \%$ & \multicolumn{2}{c|}{ Leg length } \\
\hline & Chest circumference & 0.96 & $1.92 \%$ \\
\hline 2-D system I & 0.96 & $1.77 \%$ & 0.95 & $1.97 \%$ \\
2-D system II & 0.95 & $1.81 \%$ & 0.97 & $2.89 \%$ \\
2-D system III & 0.95 & $2.37 \%$ & 0.96 & $2.13 \%$ \\
2-D system IV & 0.90 & $4.43 \%$ & & \\
\hline & Waist circumference & & \\
\hline 2-D system I & 0.98 & $1.66 \%$ & & \\
2-D system II & 0.96 & $1.78 \%$ & & \\
2-D system III & 0.97 & $3.29 \%$ & & \\
2-D system IV & 0.88 & $4.58 \%$ & & \\
\hline
\end{tabular}

In first experiment results, the Pearson correlation coefficient show that the closely perfect positive correlations were calculated and exhibited these two measurement data have similar. The results of average error rate were less than $2.5 \%$, and showed the perfect accuracy of $2 \mathrm{D}$ image-based measurement.

The second experiment performed to compare the 2D image-based measurement for each type of garment. Although the challenge of four type of garment was added in this 
experiment, the results of Pearson correlation coefficient exhibit the highly positive correlation of 2D image-based measurement and manual measurement. No matter what type of garment was worn, the average error rates of each dimension are less than $5 \%$. It can be proved that the compensation system is feasible to reduce the influence of garment thickness on measurement.

\section{B. The demonstrate of Virtual mannequins deformation}

In human body segmentation, the body is separated into eleven segments and each segment has it individual deformation that belongs to the ratio $\mathrm{Cr}$. But in our 2-D image anthropometric measurement the chest circumference, waist circumference, and hip circumference can be attained, but the significant value right and left upper and lower arm, thigh, and shank circumferences are not available. In order to create a complete virtual mannequin, these anthropometric measurement data are acquired from the manual direct measurement. Fig. 8(b) is a created virtual mannequin with the anthropometric measurement data in TABLE III, and Fig. 8(b) is the standard model at the cluster of meal at BMI range $(25 \leq$ $\mathrm{BMI}<30)$.

TABLE III. THE ANTHROPOMETRIC MEASUREMENT DATA OF STANDARD MODEL AND CREATED MODEL.

\begin{tabular}{|c|c|c|c|c|c|c|c|}
\hline$(\mathrm{cm})$ & Height & Weight & BMI & $\begin{array}{c}\text { Right } \\
\text { upper } \\
\text { arm }\end{array}$ & $\begin{array}{c}\text { Right } \\
\text { lower } \\
\text { arm }\end{array}$ & $\begin{array}{c}\text { Left } \\
\text { upper } \\
\text { arm }\end{array}$ & $\begin{array}{c}\text { Left } \\
\text { lower } \\
\text { arm }\end{array}$ \\
\hline Std. & 172 & 74.8 & 25.28 & 31.5 & 29 & 31.4 & 29.4 \\
\hline Created & 174 & 78.4 & 25.89 & 32 & 29.5 & 31.8 & 29.9 \\
\hline & $\begin{array}{c}\text { Chest } \\
\text { cir. }\end{array}$ & $\begin{array}{c}\text { Waist } \\
\text { cir. }\end{array}$ & $\begin{array}{c}\text { Hip } \\
\text { cir. }\end{array}$ & $\begin{array}{c}\text { Right } \\
\text { thigh }\end{array}$ & $\begin{array}{c}\text { Left } \\
\text { thigh }\end{array}$ & $\begin{array}{c}\text { Right } \\
\text { shank }\end{array}$ & $\begin{array}{c}\text { Left } \\
\text { shank }\end{array}$ \\
\hline Std. & 94.3 & 91.2 & 99.2 & 52.6 & 52.4 & 39.5 & 39 \\
\hline Created & 97.2 & 92.3 & 99.7 & 53.2 & 53 & 41 & 41.5 \\
\hline
\end{tabular}

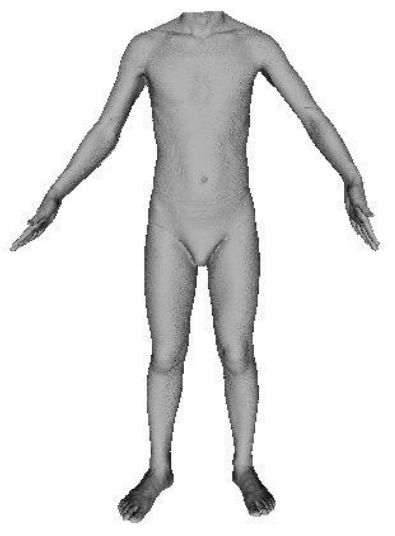

(a)

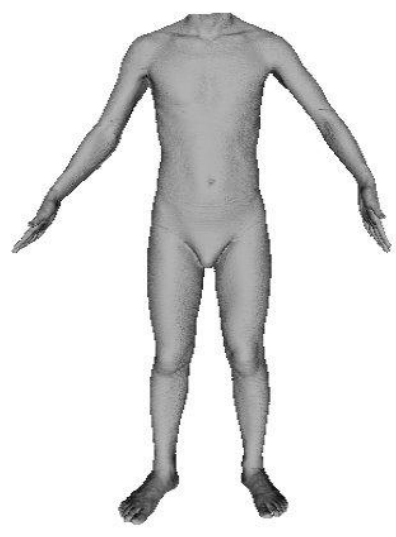

(b)
Fig. 8 demonstrates (a) the standard model and (b) The created virtual mannequin.

\section{CONCLUSION}

In this paper, we introduce a 2-D image-based anthropometric measurement system to cut out the human body silhouette and calculate the direct distances and circumferences. This method provides a more friendly measurement environment, because the conditions of subject for measurement are less than other attempts. The RD-FFD provides a fast and efficient method to create a virtual mannequin, and the example-based deformation reduces the incidence of the distortion. The cylinder coordinate also provide a good geometric space for RD-FFD. The goal of this thesis is to propose a system that have an easy and precise anthropometric measurement, and create a virtual mannequin by using the anthropometric measurement data. In the future work, the other circumference measurement will be done and the automatic segmentation and conjunction are the new challenge to take.

\section{REFERENCES}

[1] J. A. Roebuch, Jr., Anthropometric Methods: Designing to Fit the Human Body. Santa Monica, CA: Hum. Factors and Ergonom. Soc., 1995.

[2] C. C. Gordon, T. Churchill, C. E. Clauser, B. Bradtmiller, J. T. McConville, I. Tebbetts, and R. A. Walker, 1988 anthropometric survey of US army personnel: methods and summary statistic. NATICK/TR89/044. US Army Natick Research, Development, and Engineering Center, Natick, MA, 1989.

[3] P. Meunier, and S. Yin, "Performance of a 2D image-based anthropometric measurement and clothing sizing system," Applied Ergonomics, vol. 31, pp. 445-451, October 2000.

[4] C. Y. Hung, P. Witana, and S. Goonetilleke, "Anthropometric measurements from photographic images," 7th Int. Proc. on Work with computing system, pp. 104-109, June 2004.

[5] E. Paquet and H. L. Viktor, "Adjustment of virtual mannequins through anthropometric measurements, cluster analysis, and content-based retrieval of 3-D body scans," IEEE Trans. on Instrumentation and Measurement, vol. 56, pp.1924 - 1929, October 2007.

[6] K. Robinette, H. Daanen, and E. Paquet, "The CAESAR project: A 3-D surface anthropometric survey," 2nd Int. Conf. on 3-D digital imaging and modeling, Ottawa, Ont. , Canada, pp. 380-386, October 1999.

[7] D. Burnsides, M. Boehmerk, and K. Robinette, "3-D landmark detection and identification in the CAESAR project," 3rd Int. Conf. on 3-D Digital imaging and modeling, Quebec City, Que., Canada, pp. 393-398, May 2001.

[8] H. Seo and N. Magnenat-Thalman. An example-based approach to human body manipulation. Graph. Models, vol. 66, no. 1, pp. 1-23, 2004.

[9] J. Li and Y. wang. Automatically construct skeletons and parametric structures for polygonal human bodies. Computer Graphics International, 2007.

[10] A. Barr., Superquadrics and angle-preserving transformations. IEEE Computer Graphics and Applications, vol. 1, no. 1, pp. 11-23, 1981.

[11] F. Scheepers, R. E. Parent, W. E. Carlson, and S. F. May. Anatomybased modeling of the human musculature. In SIGGRAPH '97: Proceedings of the 24th annual conference on Computer graphics and interactive techniques, pp. 163-172, New York, NY, USA, 1997.

[12] P. Borrel and A. Rappoport. Simple constrained deformations for geometric modeling and interactive design. ACM Trans. Graph., vol. 13, no. 2, pp. 137-155, 1994.

[13] S. F. Lin, S. C. Chien, and K. Y. Chiu, "The 2D Image-Based Anthropologic Measurement by Using Chinese Medical Acupuncture and human body slice model," International Journal of Computer Science and Information Security," vol. 8, no. 8, pp. 20-29, Nov. 2010.

[14] J. F. David and K. Y. Yan, "Automatic image segmentation by integrating color-edge extraction and seeded region growing," IEEE Trans. on image processing, vol. 10, pp. 1454-1466, October 2001.

[15] A. Albiol, L. Torres, and E. J. Delp, "Optimum color spaces for skin detection," IEEE Int. Conf. Image processing, Thessaloniki , Greece, vol. 1, pp. 122-124, October 2001.

[16] P. J. Shen and K. W. Wu, Massage for pain relief: A step-by-step guide, morning star, Taipei, 2003. 
[17] A. Watt and H. Watt, Advanced animation and rendering techniques: theory and practice, Addison Wesley, NY, 1992.

[18] Taiwan Human Body Bank http://3d.cgu.edu.tw/DesktopDefault.asp, accessed September. 2009.

[19] C. Y. Yu, Y. H. Lo and W. K. Chiou, "The 3D scanner for measuring body surface area: a simplified calculation in the Chinese adult," Applied Ergonomics, Vol.34, pp.273-278, 2003.

[20] A. Chamberland, R. Carrier, F. Forest, and G. Hachez, Defence and civil institute of environmental medicine, Toronto, Ontario, 1997.

\section{AUTHORS PROFILE}

Sheng-Fuu Lin was born in Tainan, R.O.C., in 1954. He received the B.S. and M.S. degrees in mathematics from National Taiwan Normal University in 1976 and 1979, respectively, the M.S. degree in computer science from the University of Maryland, College Park, in 1985, and the $\mathrm{Ph} . \mathrm{D}$. degree in electrical engineering from the University of Illinois, Champaign, in 1988. Since 1988, he has been on the faculty of the Department of Electrical and Control Engineering at National Chiao Tung University, Hsinchu, Taiwan, where he is currently a Professor. His research interests include image processing, image recognition, fuzzy theory, automatic target recognition, and scheduling.

Shih-Che Chien was born in Chiayi, R.O.C., in 1978. He received the B.E. degree in electronic engineering from the Nation Chung Cheng University, in 2002. He is currently pursuing the M.E. and Ph.D. degree in the Department of Electrical and Control Engineering, the National Chiao Tung University, Hsinchu, Taiwan. His current research interests include image processing, image recognition, fuzzy theory, 3D image processing, intelligent transportation system, and animation. 